Research/Technical Note

\title{
Prevalence of Highly Multi-Drug Resistant Salmonella Fecal Carriage Among Food Handlers in Lower Basic Schools in The Gambia
}

\author{
Abou Kebbeh ${ }^{1,}$, , Bryan Anderson ${ }^{2}$, Haruna S. Jallow ${ }^{1}$, Olliemattou Sagnia ${ }^{1}$, Joseph Mendy ${ }^{1}$, \\ Yaya Camara $^{3}$, Saffiatou Darboe ${ }^{4}$, Sana M. Sambou ${ }^{3}$, Ignatious Baldeh ${ }^{1}$, Bakary Sanneh ${ }^{1}$ \\ ${ }^{1}$ National Public Health Laboratories, Ministry of Health and Social Welfare, Kotu Layout, Kotu, The Gambia \\ ${ }^{2}$ College of Public Health, The University of Iowa, Iowa, United States of America \\ ${ }^{3}$ Epidemiology and Disease Control Department, Ministry of Health and Social Welfare, Kotu Layout, Kotu, The Gambia \\ ${ }^{4}$ Department of Microbiology, Medical Research Council Unit, Fajara, The Gambia
}

Email address:

kebbehabou@yahoo.com (A. Kebbeh)

${ }^{*}$ Corresponding author

\section{To cite this article:}

Abou Kebbeh, Bryan Anderson, Haruna S. Jallow, Olliemattou Sagnia, Joseph Mendy, Yaya Camara, Saffiatou Darboe, Sana M. Sambou Ignatious Baldeh, Bakary Sanneh. Prevalence of Highly Multi-Drug Resistant Salmonella Fecal Carriage Among Food Handlers in Lower Basic Schools in The Gambia. International Journal of Nutrition and Food Sciences. Vol. 6, No. 1, 2017, pp. $39-44$.

doi: 10.11648/j.ijnfs.20170601.17

Received: December 6, 2016; Accepted: December 26, 2016; Published: January 23, 2017

\begin{abstract}
Background: Salmonella spp are among the most common food borne pathogens. Food handlers play an important role in the production of food products, in which they can contribute to the transmission of salmonellosis. The probability of food contamination depends mainly on the health status and personal hygiene of the food handlers and their choice of managing their health could give rise to contamination of food by multi drug resistant bacteria. Multi Drug Resistance is of global concern and poses a public health threat in combating diseases. In the developing countries there is paucity on the prevalence of salmonella carriage among food handlers. This study aims to investigate the prevalence of salmonella and to determine their antimicrobial resistance pattern. Method: A total of 500 stool samples from different food handlers were collected and analyzed with completed questionnaires. For standard isolation and identification of salmonella isolates; stool samples were enriched in buffered peptone water, standard culture and biochemical tests were used. Antimicrobial susceptibility Test (AST) was carried out using Clinical Laboratory and Standard Institute (CLSI-2015) protocol disc diffusion method. The data were analyzed using SPSS version 16 and Microsoft excel version 2010 to determine the risk factors. Results: Of 500 participants with the mean age of 38.15, 497(99.4\%) were all females. Most of the participants wash their hands under running water and 271(54.2\%) were certified on food handling. Among the risk factors, consuming medicines/antibiotics from street vendors showed statistical significant of salmonella carriage with $(\mathrm{P}=0.011)$. It was found that $13(2.6 \%)$ were salmonella carriers. AST performed on the 13 isolates show that; $13(100 \%), 12(92.3 \%), 7(53.8 \%), 9(69.2 \%)$ and 9(69.2\%) were resistant to Ampicillin, Erythromycin, and Tetracycline, ceftriaxone and cefotaxime respectively. And 13(100\%), 12(92.3\%) and 7(53.8) were sensitive to Imipenem, chloramphenicol and gentamycin respectively. Conclusion: This study had found multidrug resistant salmonella isolates carriers amongst food handlers who could serve as potential reservoirs for the transmission of these infections in the communities. Thus, it is crucial to implement regular screening of food handlers and health education on food safety.
\end{abstract}

Keywords: Antimicrobial Susceptibility Test, Food handlers, Salmonella, The Gambia 


\section{Background}

Salmonella is a genus of gram negative, non-spore forming, facultative anae

robic bacteria belonging to the Enterobacteriaceae family [1] The major species of concern to humans is Salmonella enterica which has many serovars that are categorized as typhoidal or non-typhoidal varieties. Several thousand non-typhoidal serovars exist with the predominant strains varying from region to region but the most common serovars being Salmonella enteritidis ( $S$. enteritidis) and Salmonella typhimurium ( $S$. Typhimurium) which comprise approximately three-quarters of the global yearly cases of salmonellosis [2] [3]. Typhoidal Salmonella, divided into serovars Salmonella typhi (S. typhi) and Salmonella paratyphi (S. paratyphi), as the names suggest, are the cause of typhoid fever [1].

Non-typhoidal salmonellae (NTS) are responsible for causing a gamut of illnesses ranging from diarrheal diseases, bloodstream infections, and meningitis [4] [5]. Diarrheal NTS is the main contributor of foodborne illness and remains a public health concern worldwide [2]. Those afflicted with NTS can be asymptomatic carriers that continue to shed the bacteria or have varying severity of clinical symptoms such as diarrhea, vomiting, and other gastrointestinal problems [1].

Transmission of Salmonella is usually the result of poor hygiene during food handling with approximately $70 \%$ of diarrheal diseases being the result of contaminated food in the developing world [13], [14]. Improper handwashing after using toilet facilities, lack of education for handling food and cross contamination are all identified sources of likely salmonella transmission [6]. Animal sources are the most common source of NTS which is carried in the intestinal tract of food animals, intestinal contents of NTS infected animals often times contaminate the meat during the butchering process and is spread to food preparation surfaces and utensils [15]. Typhoidal salmonella has very few animal hosts and is the result of fecal-oral transmission of the bacteria from person-to-person [14]. Additionally, asymptomatic carriers of NTS and typhoidal Salmonella continue to shed bacteria in their feces and urine providing an opportune pathway of infection for the pathogen [14].

On a global scale, foodborne illness afflicts an estimated $30 \%$ of the population with Salmonella commonly attributed as one of the top 5 pathogens [7] [8]. According to the WHO using 2010 statistics, NTS is of particular concern in Africa where $54 \%$ of the deaths from NTS occurred [9]. Across eastern Africa, the prevalence of Salmonella varies by region. In Ethiopia, several studies have been performed looking at food handlers at university campuses. At Bahir Dar University, $2.7 \%$ of the food handlers tested positive for S. Typhi [7]. Additionally, studies performed amongst food handlers in Ethiopia in the southern region, University of Gondar, dairy workers, and Addis Abbaba University showed prevalence of $3.1 \%, 0.93 \%, 5.0 \%, 4.8 \%$ respectively [2] [6] [10] [11]. A 2015 study by David et al at a Nigerian university showed a prevalence of 35\% amongst food handlers following a recent outbreak of typhoid fever [12]. In western Africa, a study published in 2016 took random samples from households in Bissau, Guinea-Bissau and Dakar, Senegal. Results showed that no $S$. typhi was isolated but NTS was cultured across all age ranges. In Dakar there was a prevalence of 10.3/1000 population and in Bissau a prevalence of 24.1/1000 population with children ages 5-14 having 4 times higher rates of excretion in Guinea-Bissau than in Senegal [1]

In The Gambia, Salmonella cultures were analyzed from samples taken between 2005 and 2015 from medical clinics. Among the samples, 14\% were found to have $S$. typhi and the remaining $86 \%$ were found to have various serovars of NTS including $S$. Typhimurium, $S$. Enteritidis, and $S$. Arizonae [5].

However, wide spread Multi Drug Resistant (MDR) is of a global concern due to the difficulty in treating and potential for dissemination in an increasingly mobile global culture [10]. This poses a public health threat and has great implication on economics and patient care. Garedew et al reported in their studies that 13 isolates of Salmonella are resistance to various antibiotics, amoxicillin, ampicillin, nitrofurantoin and tetracycline and half of the isolates were MDR [10]. The study by $\mathrm{Im}$ et al in Senegal and Guinea-Bissau, varying antibiotic resistance was detected [5]. In Guinea-Bissau, 7 of the 22 Salmonella samples were resistant to ampicillin and in Senegal, 3 of the 14 Salmonella isolates were resistant to cotrimoxazole [1]. In The Gambia, Kwambana et al reports, 4\% (9/203) of Salmonella isolates had MDR, showing that MDR exists in Salmonella in The Gambia [5]. Overuse of antimicrobials, medical professionals prescribing antibiotics without resistance testing, prolonged hospitalizations and their related infections, and issues of patient self-medication are all potentially driving the trend of increased MDR [10]. However, with emerging of MDR salmonella, its study among possible carriers (food handlers) in developing countries like The Gambia is limited. This current study aims to investigate the prevalence of salmonella among food handlers and determine their antimicrobial susceptibility resistance pattern.

\section{Methodology}

\subsection{Study Design}

This current study was carried out among food handlers in lower basic schools in the West Coast Region of the country. Sample size was determined by following a simple formula (Daniel, 1999) $Z^{2} P(1-P) / d^{2}$ considering the following assumption $Z=1.96$, precision $\mathrm{P}=5 \%$ and standard scale of $95 \%$ confidence interval $(\mathrm{CI})$.

\subsection{Data Collection and Sample Procedure}

This current study samples and data were extracted from the cross sectional study Extended Spectrum Beta Lactamase 
among food handlers, which was approved by the Gambia Government and Medical Research Council Joint ethical committee with a number SCC1416. Written informed consent was obtained from all the enrolled food handlers by their signatures or fingerprints. A total of 500 food-handlers participants with complete data were enrolled in the study. Samples were collected randomly from consented participants Stool samples were collected in suitably labeled leak-proof plastic containers using clean wooden applicator stick. Samples were immediately transported to the reference laboratory using an icebox. The samples were stored at $-32^{\circ} \mathrm{C}$ until further investigation.

\subsection{Laboratory Investigation}

Out of the 600 food handlers that participated, 500 stool samples with complete data were analyzed in this current study. Salmonella isolation was done by using Clinical Laboratory Standard Institute (CLSI-2015) guidelines [16]. Approximately $1 \mathrm{~g}$ of stool sample were inoculated in $10 \mathrm{ml}$ of buffered peptone water enrichment medium and incubated for $24 \mathrm{hrs}$ at $37^{\circ} \mathrm{C}$ [4]. After incubation, $100 \mu \mathrm{l}$ of the inoculated enrichment broth was cultured on Xylose Lysine Deoxycholate (XLD)/MacConkey. Suspected non-lactose fermenter colonies were purified using Muller Hinton agar and incubated for $24 \mathrm{hrs}$ at $37^{\circ} \mathrm{C}$. Purified colonies were subjected to biochemical tests.

\subsection{Biochemical Tests}

Presumptive isolates of Salmonella spp were confirmed with the following tests; oxidase, catalase, Voges Proskauer (VP), Indole, Simmons Citrate and Tripple Sugar Iron and the $\mathrm{H}_{2} \mathrm{~S}$ gas production. Isolates those are negative for indole, oxidase and Voges Proskauer, and positive for catalase,
Simmons Citrate, Tripple Sugar Iron and $\mathrm{H}_{2} \mathrm{~S}$ gas production were confirmed as salmonella isolates.

\subsection{Antibiotic Susceptibility}

Antimicrobial susceptibility test were done on all the confirmed isolates using Muller Hinton agar in accordance with CLSI protocol disk diffusion method [16]. The following antibiotics were used; Ampicillin, Erythromycin, Chloramphenicol, Ciprofloxacin, Nitrofurantoin, Tetracycline, Cefotaxime, Cotrimazole, Ceftoxitin, Ceftriaxone, Gentamycin, and Imipenem. The zone of inhibition was measured in accordance to CLSI-2015 protocol, results were interpreted as resistant, intermediate or susceptible as recommended by CLSI-2015.

\subsection{Data Analysis}

Data analyses were done using software SPSS version 16.0 and Microsoft Excel version 2010. The chi-square test was used to determine possible association of salmonella isolates with socio-demographic and risk factors. P value $<0.05$ was set as statistical significant.

\section{Results}

\subsection{Socio-demographic of the Study Participant}

Overall, 500 food handlers were enrolled in this study with the mean age of 38.15 years, of which $497(99.4 \%)$ were females. Most of the participant lived less than $5 \mathrm{~km}$ away from a health facility $490(98 \%)$. Of the total participant, $271(54.2 \%)$ were certified for food handling, $90.2 \%$ know the principle of food safety and $41.6 \%$ were trained on food handling, as shown in table 1.

Table 1. Demographic information.

\begin{tabular}{|c|c|c|c|}
\hline Demographic information & Outcome & $\%$ & P-Value \\
\hline Female gender & 497 & 99.4 & 0.777 \\
\hline Mean age (years) & 38.15 & & \\
\hline Staying in homes $<5 \mathrm{~km}$ from health facility & 490 & 98 & 0.137 \\
\hline Is there any domestic animals in your home? & 297 & 59.4 & 0.874 \\
\hline Certified for food handling & 271 & 54.2 & 0.163 \\
\hline Do you know the principle of food safety? & 451 & 90.2 & 0.229 \\
\hline Trained on food handling & 208 & 41.6 & 0.917 \\
\hline
\end{tabular}

\subsection{Risk Factors Associated with Salmonella Carriage}

Almost all the food handlers claim of washing their hands with soap under running water 498(99.6\%). In addition, all of them used stand pipe as a source of drinking water. Out of 500 participants, $86(17.2 \%)$ were sick and admitted in hospital in the past three months. However, statistical analysis of salmonella carriage versus hand washing with soap under running water showed no significant with $\mathrm{P}>0.05$. Statistical analysis between salmonella isolates and consuming antibiotic from street vendors showed a significant association ( $\mathrm{P}$ value 0.011 ). Additionally, it was found that, up to $84.6 \%$ (11 out of 13) of the isolates were recovered from certified food handlers, as shown in the table 2 .

Table 2. Data on Risk factors for salmonella fecal carriage.

\begin{tabular}{|c|c|c|c|}
\hline Risk factors & number of respondent & $\%$ & P-Value \\
\hline Do you normally wash hands with soap under running water? & 498 & 99.6 & 0.85 \\
\hline Sick and admitted in hospital in the last three months & 86 & 17.2 & 0.393 \\
\hline Do you have history of medical instrumentation while on admission? & 4 & 0.8 & 0.743 \\
\hline Have ever escorted/visited a sick family member to the hospital in the last three (3) months? & 70 & 14 & 0.507 \\
\hline
\end{tabular}




\begin{tabular}{llll}
\hline Risk factors & number of respondent & \% & P-Value \\
\hline Have you ever stayed with somebody who was admitted in the hospital long/short term? & 87 & 17.4 & 0.35 \\
Have you taken any antibiotics in the last three (3) months? & 168 & 33.6 & 0.707 \\
Did you ever buy and consume medicines/antibiotics from street vendors? & 16 & 3.2 & 0.011 \\
Did you complete taking your prescribed antibiotics? & 482 & 96.4 & 0.48 \\
Did you have diarrhoea in the last three months? & 52 & 10.4 & 0.213 \\
Did you experience urinary pain during urinating in the last three (3) months? & 10 & 2 & 0.137 \\
\hline
\end{tabular}

\subsection{Salmonella Isolates}

Salmonella were isolated from 13 food handlers out of the 500 food handlers screened; giving $2.6 \%$ salmonella carriage. All the isolates were from female participants.

\subsection{Antimicrobial Susceptibility Profile of Salmonella Isolates}

All the salmonella isolates were subjected to antimicrobial susceptibility test with 12 different antibiotics. All isolates were resistant to ampicillin 13(100\%). Overall, 12(92.3\%) of the isolates were resistant to erythromycin and only $1(7.7 \%)$ isolate was intermediate susceptible. More than half of the isolates were resistant to both Cefotaxime and ceftriaxone that is $9(69.2 \%)$, whilst $4(30.8 \%)$ were susceptible to both. Only $2(15.4 \%)$ isolates were resistant to ciprofloxacin and
10(76.9\%) were intermediate susceptible. Additionally, more than half $7(53.8 \%)$ of the isolates were resistant to tetracycline and $2(15.4 \%), 4(30.8 \%)$ were sensitive and intermediate susceptible respectively. Moreover, at least three quarter of the isolates were susceptible to cotrimazole; 6(46.2\%) and $2(15.4 \%)$ were sensitive and intermediate susceptible respectively. On the other hand, all the isolates were susceptible to Imipenem and chloramphenicol; 13(100\%) and $12(92.3 \%)$ respectively with $1(7.7 \%)$ intermediate susceptible to chloramphenicol. Additionally, more than half 7(53.8\%) of the isolates were sensitive susceptible to both ceftoxitin, nitrofurantoine and gentamycin, and 6(46.2\%) were intermediate susceptible to gentamycin. However, none of the salmonella isolates were sensitive to all antimicrobial agent used, as shown in table 3.

Table 3. Antibiotic resistance pattern of the salmonella isolates.

\begin{tabular}{|c|c|c|c|c|c|c|}
\hline \multirow{3}{*}{ Antibiotics } & \multicolumn{6}{|c|}{ Resistance Patterns } \\
\hline & \multicolumn{2}{|c|}{ SENSITIVE } & \multicolumn{2}{|c|}{ INTERMEDIATE } & \multicolumn{2}{|c|}{ RESISTANT } \\
\hline & $\mathbf{N}$ & $\%$ & $\mathbf{N}$ & $\%$ & $\mathbf{N}$ & $\%$ \\
\hline cefotaxime $30 \mu \mathrm{g}$ & 4 & 30.8 & 0 & 0 & 9 & 69.2 \\
\hline Ceftriaxone $30 \mu \mathrm{g}$ & 4 & 30.8 & 0 & 0 & 9 & 69.2 \\
\hline Cefotoxitin $30 \mu \mathrm{g}$ & 7 & 53.8 & 2 & 15.4 & 4 & 30.8 \\
\hline Imipenem $10 \mu \mathrm{g}$ & 13 & 100 & 0 & 0 & 0 & 0 \\
\hline Nitrofurantoin $300 \mu \mathrm{g}$ & 7 & 53.8 & 1 & 7.7 & 5 & 38.5 \\
\hline Ciprofloxacin $10 \mu \mathrm{g}$ & 1 & 7.7 & 10 & 76.9 & 2 & 15.4 \\
\hline Erythromycin $10 \mu \mathrm{g}$ & 0 & 0 & 1 & 7.7 & 12 & 92.3 \\
\hline Ampicillin $10 \mu \mathrm{g}$ & 0 & 0 & 0 & 0 & 13 & 100 \\
\hline Tetracycline $10 \mu \mathrm{g}$ & 2 & 15.4 & 4 & 30.8 & 7 & 53.8 \\
\hline Cotrimazole $30 \mu \mathrm{g}$ & 6 & 46.2 & 2 & 15.4 & 5 & 38.4 \\
\hline Gentamycin $10 \mu \mathrm{g}$ & 7 & 53.8 & 6 & 46.2 & 0 & 0 \\
\hline Chloramphenicol $30 \mu \mathrm{g}$ & 12 & 92.3 & 1 & 7.7 & 0 & 0 \\
\hline
\end{tabular}

\section{Discussion}

The current study showed $2.6 \%$ of salmonella carriage among food handlers which is consistent with $2.7 \%$ prevalence reported from Ethiopia [7]. The salmonella positive participants in this current study were all females as observed in a study in Ethiopia by Getnet et al [6]. The overall carriage of salmonella in the current study shows similar result as reported in University of Gondar, Ethiopia, 3.1\% [10]. This rate of carriage contrast with a rate of $31.4 \%$ of salmonella enterica serovar and paratyphi infections among food handlers in Lagos Nigeria Ogah et al and in a study from Karachi-Pakistan showing overall high prevalence of salmonella 9.1\% [8] [17]. Salmonella infection in The Gambia, shows high prevalence of $39 \%$ of the analyzed stool specimens from hospitalized patients; which is not in agreement with our findings among food handlers [5]. This dissimilar findings may be due to the method of sampling as study by Kwambana et al centered on patients hospitalized and we centered our sampling on the community. However, this rate is much higher compare to a study in Ethopia where one isolate of salmonella was isolated out of 107 food handlers tested and in Jordan where no single salmonella 
isolate was identified [11] [13].

Previous studies have reported MDR of salmonella isolates, the results of the current research have showed multi-drug resistant of most of the isolates [5] [10]. Interestingly, all the isolates were resistant to ampicillin, which concords with a finding in Ethiopia where all isolates are resistant and one intermediate susceptible [6]. In addition, 7(53.8\%) were resistant to both tetracycline and nitrofurantoine. All isolates were sensitive to gentamycin with $7(53.8 \%)$ susceptible and $6(46.2 \%)$ intermediate susceptible these findings were in agreement with Garedew et al. [10]. In the current study, $9(69.2 \%)$ resistant to cephalosporins (ceftriaxone and cefotaxime) were comparable to a study in Pakistan which recorded 5.2\% [17]. This contrast the findings of Garedew et al where all the isolates were susceptible to ceftriaxone, this may be due to the high utilization of antibiotics from street vendors. Furthermore, a study in Senegal reported that all the 14 isolates were susceptible to chloramphenicol which concords with our findings, where $12(92.3 \%)$ were sensitive susceptible and $1(2.7 \%)$ intermediate susceptible. However, in the same study 3 of 14 isolates were resistant to cotrimaxole which shows a lower rate compared to our findings [1]

\section{Conclusion}

This study revealed the Salmonella isolates that are drug resistant including MDR isolates that are in circulation in the community among food handlers. The current study also found the relation of consuming medications from street vendors and harboring the MDR salmonella. The Salmonella carriers can continue to shed the bacteria and infect the population specially the students at lower basic schools. Thus, it is crucial to implement regular screening of food handlers and health education on food safety and alert people about drug resistance and the impact of misusing drugs.

\section{Acknowledgement}

The authors wish to acknowledge the study team of ESBL project for allowing us to use their banked samples and data. We wish to acknowledge WHO Department of HSE/FOS for funding the ESBL project which was given a funding number SPHQ14-APW-3979. The authors are grateful to Mr. Alieu Faal, Mr. Ebrima Joof and Abdoulie M. Sanyang for their effort in reviewing the work. We wish to acknowledge the study participants for their willingness and interest to participate. We acknowledge the Ministry of Health for the technical advices and uses of laboratory resources.

\section{References}

[1] J. Im, C. Nichols, M. Bjerregaard-Andersen, A. G. Sow, S. Løfberg, A. Tall, G. D. Pak, P. Aaby, S. Baker, J. D. Clemens, L. M. C. Espinoza, F. Konings, J. May, M. Monteiro, A. Niang, U. Panzner, S. E. Park, H. Schütt-Gerowitt, T. F. Wierzba, F. Marks, and V. Von Kalckreuth, "Prevalence of Salmonella Excretion in Stool: A Community Survey in 2 Sites,
Guinea-Bissau and Senegal," Clin. Infect. Dis., vol. 62, no. Suppl 1, pp. s50-s55, 2016.

[2] M. M. Gwida and M. A. M. Al-Ashmawy, "Culture versus PCR for salmonella species identification in some dairy products and dairy handlers with special concern to its zoonotic importance," Vet. Med. Int., vol. 2014, 2014.

[3] R. S. Hendriksen, A. R. Vieira, S. Karlsmose, D. M. a Lo Fo Wong, A. B. Jensen, H. C. Wegener, and F. M. Aarestrup, "Global monitoring of Salmonella serovar distribution from the World Health Organization Global Foodborne Infections Network Country Data Bank: results of quality assured laboratories from 2001 to 2007.," Foodborne Pathog. Dis., vol. 8, no. 8, pp. 887-900, 2011.

[4] N. A. Feasey, G. Dougan, R. A. Kingsley, R. S. Heyderman, and M. A. Gordon, "Invasive non-typhoidal salmonella disease: An emerging and neglected tropical disease in Africa," Lancet, vol. 379, no. 9835, pp. 2489-2499, 2012.

[5] B. Kwambana-Adams, S. Darboe, H. Nabwera, E. Foster-Nyarko, U. N. Ikumapayi, O. Secka, M. Betts, R. Bradbury, R. Wegmüller, B. Lawal, D. Saha, M. J. Hossain, A. M. Prentice, B. Kampmann, S. Anderson, U. Dalessandro, and M. Antonio, "Salmonella infections in The Gambia, 2005-2015," Clin. Infect. Dis., vol. 61, no. Suppl 4, pp. S354-S362, 2015.

[6] F. Getnet, S. Gebre-selassie, H. Alemayehu, T. Kassa, and N. Kebede, "Prevalence and Antimicrobial Resistance of Salmonella Isolated from Food Handlers in Addis Ababa University Students 'Cafeteria, Ethiopia," vol. 6, no. 6, pp. 210-216, 2014.

[7] B. Abera, G. Yitayew, and H. Amare, "Salmonella serotypetyphi, shigella, and intestinal parasites among food handlers at bahir dar university, Ethiopia," J. Infect. Dev. Ctries., vol. 10, no. 2, pp. 121-126, 2016.

[8] J. M. M. Diagn, O. Jo, A. Oc, and A. Aa, "Medical Microbiology \& Diagnosis Prevalence of Salmonellosis among Food Handlers and the Health Implications on the Food Consumers in Lagos State, Nigeria," vol. 4, no. 2, pp. 4-8, 2015.

[9] A. H. Havelaar, M. D. Kirk, P. R. Torgerson, H. J. Gibb, T. Hald, R. J. Lake, N. Praet, D. C. Bellinger, and N. R. De Silva, "World Health Organization Global Estimates and Regional Comparisons of the Burden of Foodborne Disease in 2010," pp. 1-23, 2015.

[10] L. Garedew-Kifelew, N. Wondafrash, and A. Feleke, "Identification of drug-resistant Salmonella from food handlers at the University of Gondar, Ethiopia.," BMC Res. Notes, vol. 7, p. $545,2014$.

[11] M. Birhaneselassie and D. Williams, "A study of salmonella carriage among asymptomatic food-handlers in southern Ethiopia," vol. 2, no. 5, pp. 243-245, 2013.

[12] O. M. David and A. O. Oluyege, "Antibiotic resistance and plasmid carriage among Salmonella typhi isolated from food and hand of food handlers in a Nigeria university.," Int. J. Curr. Microbiol. Appl. Sci., vol. 4, no. 3, pp. 906-914, 2015.

[13] M. Abdel-dayem, R. Al, R. Bani, and Z. Sami, "Microbiological and parasitological investigation among food handlers in hotels in the Dead Sea area, Jordan," J. Microbiol. Immunol. Infect., vol. 47, no. 5, pp. 377-380, 2014. 
[14] A. Gebreyesus, K. Adane, L. Negash, and T. Asmelash, "Prevalence of Salmonella typhi and intestinal parasites among food handlers in Mekelle University student cafeteria, Mekelle, Ethiopia," Food Control, vol. 44, pp. 45-48, 2014.

[15] E. Carrasco, A. Morales-Rueda, and R. M. García-Gimeno, "Cross-contamination and recontamination by Salmonella in foods: A review," Food Res. Int., vol. 45, no. 2, pp. 545-556, 2012.
[16] S. Testing, M100-S24 Performance Standards for Antimicrobial, no. January. 2015.

[17] T. R. Siddiqui, S. Bibi, M. A. Mustufa, S. M. Ayaz, and A. Khan, "High prevalence of typhoidal Salmonella enterica serovars excreting food handlers in Karachi-Pakistan: A probable factor for regional typhoid endemicity," J. Heal. Popul. Nutr., vol. 33, no. 1, pp. 1-9, 2015. 